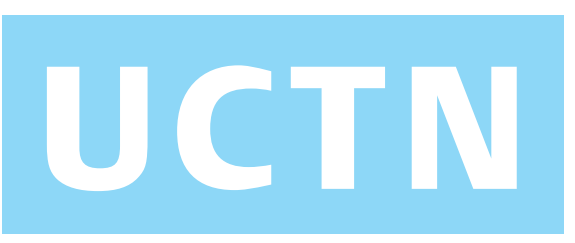

\title{
Rectal endometriosis mimicking a large polypoid rectal cancer
}

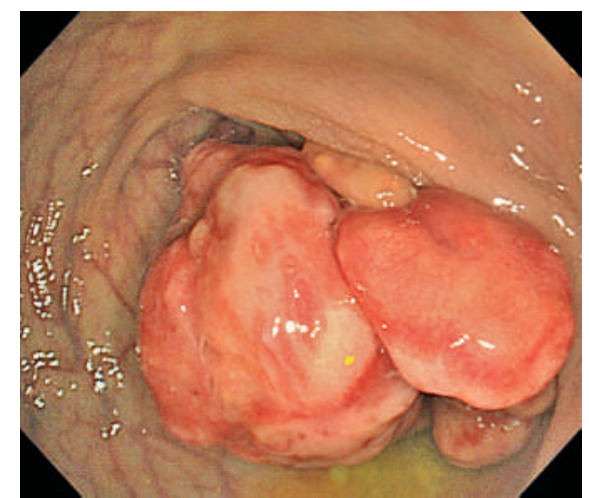

Figure 1 Colonoscopic view showing a large, ulcerating polypoid mass that was obstructing the lumen.

Endometriosis is a disorder in which endometrial glandular tissue and stroma are found outside the uterus; it involves the colorectum in about $5 \%$ of cases $[1,2]$. It can occasionally be difficult to differentiate between colonic endometriosis, usually manifest as strictures or external compression, and colon cancer or colitis [3]. In particular, the rare polypoid endometriosis is hardly distinguishable from colonic neoplasm [4]. More careful inspection and diagnostic methods are therefore required. We report a case of rectal polypoid endometriosis mimicking a large polypoid rectal carcinoma.

A 29-year-old woman presented with alteration in bowel habit and intermittently blood-tinged stools over a period of a few months. Colonoscopy revealed a large ulcerating polypoid mass $8 \mathrm{~cm}$ from the anal verge which nearly obstructed the lumen (Figure 1). We thought that this was a polypoid rectal adenocarcinoma. Computed tomography revealed an enhancing soft-tissue mass with pericolic tumor infiltration, and bilateral cystic ovarian masses. Endoscopic biopsy, which was performed on two separate occasions, showed chronic active inflammation with granulation tissue and regeneration-type cytological atypia at first, and a hamartoma at the second biopsy.

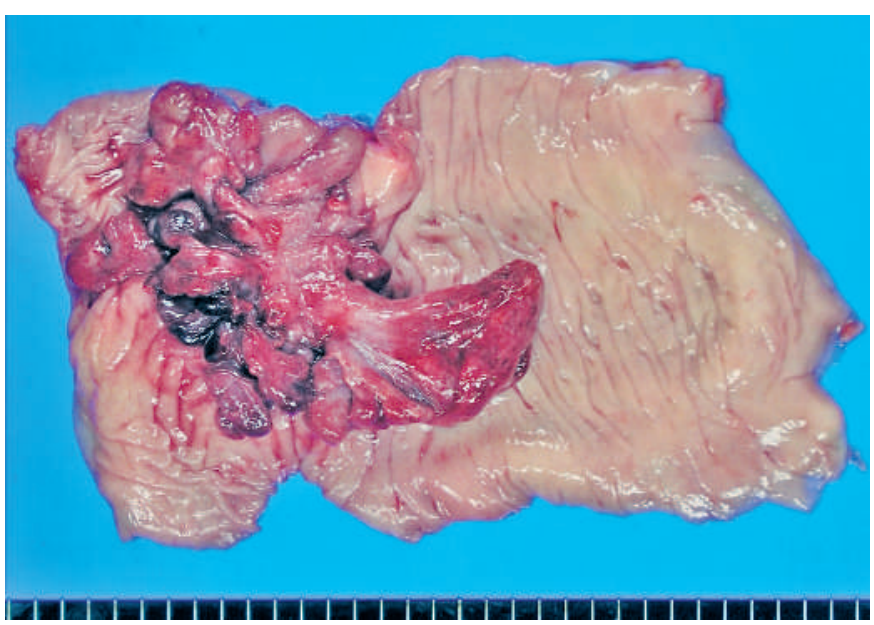

Figure 2 Grossly, the mass was found to be composed of multiple hemorrhagic grayish polyps and was attached to the rectal wall.

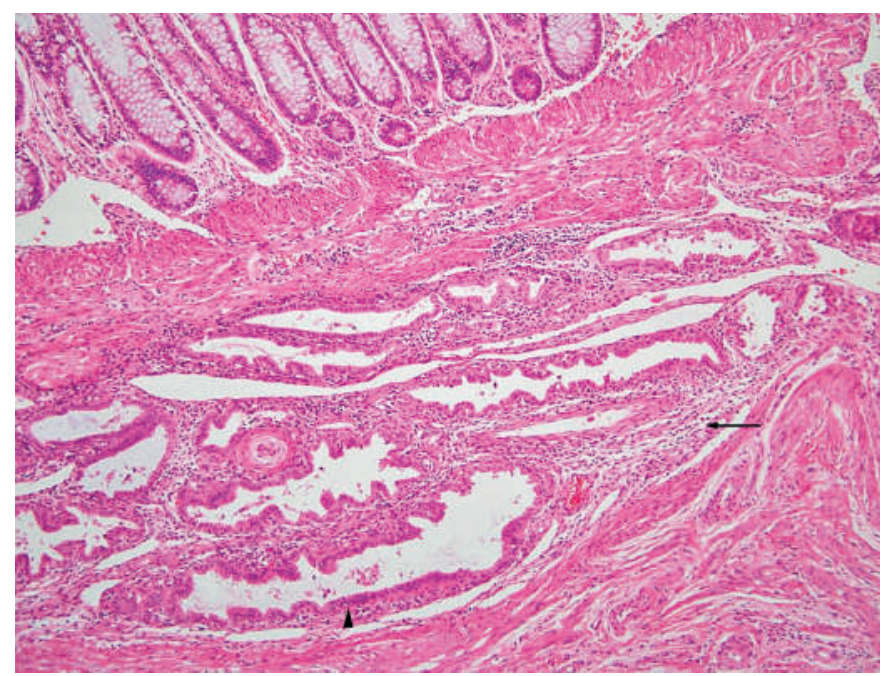

Figure 3 Histological examination of the rectal mass showed endometrial glands (arrow head) and surrounding stroma (arrow).

Because rectal cancer could not be ruled out, the patient underwent low anterior resection and cystectomy. The mass was attached to the rectal wall and was composed of multiple hemorrhagic, grayish polyps (Figure 2). Microscopic examination showed endometrial glands and surrounding stroma, and a diagnosis of rectal endometriosis was made (Figure 3). Pathological examination of the ovarian cysts showed an endometrioma on the right ovary and a corpus luteum cyst on the left. A pericolic lymph node showed reactive hyperplasia.
Although endometriosis is asymptomatic in many patients, rectosigmoid endometriosis can result in changes in bowel habit. Magnetic resonance imaging has been proved to be valuable in the diagnosis of endometriosis [1]. If the colonic mucosa is not involved, the exact diagnosis might not be made by colonoscopic biopsy [5]. Laparotomy is a therapeutic option [2].

Endoscopy_UCTN_Code_CCL_1AD_2AC 
Y. J. Cho, Y. T. Jeen, Y. S. Kim, H. J. Chun, S. H. Um, C. D. Kim, H. S. Ryu

Department of Internal Medicine, Institute of Digestive Disease and Nutrition, Korea University College of Medicine, Seoul, Korea.
References

${ }^{1}$ Olive DL, Schwartz LB. Endometriosis. N Eng J Med 1993; 328: 1759-1769

2 Jatan AK, Solomon MJ, Young J et al. Laparoscopic management of rectal endometriosis. Dis Colon Rectum 2005; 49: 1 -6

${ }^{3}$ Yantiss RK, Clement PB, Young RH. Endometriosis of the intestinal tract: a study of 44 cases of a disease that may cause diverse challenges in clinical and pathologic evaluation. Am J Surg Pathol 2001; 25: 445 - 454

${ }^{4}$ Parker RL, Dadmanesh F, Young RH et al. Polypoid endometriosis: a clinicopathologic analysis of 24 cases and a review of the literature. Am J Surg Pathol 2004; 28: $285-$ 297

${ }^{5}$ Paksoy M, Karabicak I, Ayan F et al. Intestinal obstruction due to rectal endometriosis. Mt Sinai J Med 2005; 72: 405-408
Corresponding Author

\section{Y. T. Jeen, M.D.}

Department of Internal Medicine Institute of Digestive Disease and Nutrition

Korea University College of Medicine 126-1 Anam-dong 5-ga, Seongbuk-gu Seoul 136-705

Korea

Fax: $\quad$ +82-2-953-1943

E-mail: kumcge@chol.com 